\title{
Effectiveness of Community-Based Health Education Intervention Programme on Substance Abuse among College of Education Students in Borno State, Nigeria
}

\author{
*Garba, H; Summaiya A.T, Elizabeth A.H \\ Department of Physical and Health Education, Faculty of Education, University of Maiduguri, Borno State \\ Nigeria \\ Corresponding Author: Garba, $H$
}

\begin{abstract}
This study was conducted to determine the effectiveness of Community-based Health Education Intervention Programme on substance abuse among College of Education Students in Nigeria. The study was guided by one objective, a research question and one hypothesis. The population of the study comprised the entire 3798 (2563 male and 1235 female) Nigeria certificate in Education students in College of Education Waka-Biu, Borno State Nigeria. The sampled departments include Economics and Geography from school of Arts, Mathematics and Physical and Health Education from School of Sciences, Agricultural Education and Home Economics from School of Vocational Education Primary Education and Early Child Education Department. The selected departments had a student's population of one thousand two hundred (N=1256) out of which one hundred fifty five (155)students were identified as substance abusers using 24-item substance abuse identification inventory and one hundred and five (N-105) (male54 and female 51) subjects consented to participate in the study. The reliability coefficient of the instrument was 0.89 which was found to be high enough for the study. Data collected were analyzed using descriptive statistics of mean; standard deviations and percentage to answer research questions. While inferential statistics of independent sample paired t-test, and multivariate analysis of variance were also used to test the hypothesis. Hypothesis was tested at 0.05 alpha level of significance. The result revealed that significant difference existed on the community-based health education intervention after six weeks of intervention at pretest and post test which showed that the intervention programmes were effective on knowledge of substance abuse. It was concluded that community based health education intervention programme is effective on substance abuse among students in college of education. Based on the result of this study, it was recommended that substance abuse health education intervention programme should be intensified in all our educational institutions at all levels.
\end{abstract}

Keywords: Substance Abuse, Intervention, Programme, Community Based and Health Education

Date of Submission: 12-07-2017

Date of acceptance: 24-07-2017

\section{Introduction}

Substance abuse is the use of a chemical substance which results in an individual's, physical, mental, emotional or social impairment. Substance abuse is the intake of any substance by a person that modifies perception, mood, cognition, behavior or motor functions which includes illicit and licit substances, whether or not their use brings about dependence problems in the individual or not but considered harmful. Substance abuse in Africa has increase compared with decrease in other parts of the world. Currently Africa and Asia account for $70 \%$ of global population using substances such as opium and its derivatives. Nigeria remains one of the countries with the highest prevalence rate of substance users in Africa. Epidemiological survey conducted by Federal Ministry of Education in 1995 revealed that $11 \%$ of students have abused substance such as tobacco and its derivatives, while 5\% have abused cannabis, amphetamine, cocaine, and heroin. Adam ( 1995) reported that students have abused substances ranging from stimulants to depressants and from hallucinogens to inhalant which often can be obtained from friends, medicine dealers, market stores, and mobile substance peddlers with little or no difficulties. Similarly, in Borno State according to Garba and Gaya (2005) there is a wide spread substance abuse among students. It is common to see students get intoxicated due to substance abuse, which is a major problem facing students that cut across gender and age and the most commonly abused are psychoactive substances and that psychological and academic factors are the major factors that contribute to substance abuse among the students. Consequently, according to Hyelasikida and Mbaya (2004) 78\% of students perceive substance abuse as a fashion in life, means to rebelliousness against school authority and others being ignorant of its effects and sees it as a motivating factor in school activities. Substance abuse among Colleges Students have become the order of the day due to this, many students have suffered substance abuse related problems 
such as academic failure, psychological problems, violence, rape, stealing and other social vices (Makanjuola, Daramola,\& Obembe, 2007; Eneh \&Stanly 2004). This has called for health education intervention among the students. Health education intervention programme is a heuristic device used to conceptualize and organize a large variety of activities for intervention. According to Stalker, Altman, Brawn, Burndine, Goodman and Forgersen (2012) health education intervention is one of the means in which individuals' abusive behavior can be positively influenced.

Community-based intervention refers to as an intervention programme in which community resources is used to intervention on common health issues. Accordingly, Wandersman \& Florini (2003) stated that community-based health education intervention programme is used for behavioural changes. It is a multicomponent intervention generally combined to enhance change across multiple settings with the view to promoting well being. The popularity of community-based intervention stems from its multiple roots empowerment community, capacity building and social capital for individual development (Chou, Montgomery, Pentz, Robrbach, Johson, Flay \& Mackinnon, 1998 \& Medico-social Commission, 1998).This intervention emphasized engagement from grassroots participation, increased inter-organizational linkages, strengthening community setting and problem solving. This intervention has become a popular community-driven strategy for identifying needs and developing solutions for health problems in communities. The community-based intervention is an organized human and material resource to effect specific change in their members based on issues or conditions at hand (Biglan, Ary, Smolkowski, Ducan \& Black, 2000). According to Resnicow, Soler, and Bajracharga (2009), Racha and Soarers (2009), this approach is so important to individuals in the community and is used because of its capacity to translate health messages or campaign into the setting. The interventions strategies target substance abuse for the purpose of preventing this anti-social behavior.

\section{Statement of the Problem}

The basic expectation of health education intervention programme on substance abuse is to provide knowledge on substance abuse which could lead to behavioural changes. Health education intervention programme does not only lead to behavioural changes but also to increase knowledge on substance abuse. This could be gained through the application of community-based health education intervention programmes. A well directed substance abuse health education intervention programme enhances appropriate knowledge on substance abuse that will be effective for behavioural change. In Nigeria youths tend to use some inducement drug more common as psychoactive substances. This is to enable them face their farming activities effectively. These youths seems to apply the same in every situations they find themselves, this has resulted in the lynching of security personnel and wanton destruction of lives and properties The students being a part of the larger community seems to apply the same in the school, this may be connected with the frustration resulting from academic demands in school and social stresses experienced by them Students in College are important human resources for the future development of the state and the country at large. Some of these students who are in the stage of experimentation and exploration, and their curiosity away from home are particularly prone to much destructive behaviour such as substance abuse. This situation is much more pronounced among youths aged 16 to 25 years. Despite government effort to stem the tide of substance abuse through policy formulation, establishment of National Drug Law Enforcement Agency (NDLEA) and the utilization of military, police and other paramilitary for substance abuse control, it has prove less effective. This trend has created room for the proliferation of substance addictive behaviour in the school, where aggressive lecturers and other administrative structures in the school face insults, mob action and arson. It is against this background that this study was designed to determine the effectiveness of community-based, peer-led, and school-based health education intervention programme on substance abuse knowledge among students in College of Education Waka-Biu, Borno State, Nigeria.

\section{Objective of the Study}

The study sought to determine the effectiveness of community-based health education intervention on substance abuse at pre test and post-test intervention of students in College of Education Waka-Biu, Borno State, Nigeria.

\section{Research Question}

What is the effectiveness of community-based health education intervention programme on student's knowledge of substance abuse, reasons, effects of substance abuse at pretest and post-test intervention among students in College of Education Waka-Biu Borno State Nigeria?

\section{Hypothesis}

$\mathrm{H}_{\mathrm{O} 1}$. There is no significant difference between pretest and post test scores of student's knowledge of substance abuse, reasons for substance abuse and effects of substance abuse taught using community-based health education intervention programme. 


\section{Methodology}

The pre-test, post-test quasi-experimental research design was used for this study. The use of this design was based on the premises that it is difficult to subject human participants to full laboratory control and laboratory isolation, as required in true experiments

The population of this study consisted of all 3798 (male 2563 and female1235) Nigeria Certificate in Education students. The selected departments had a student's population of one thousand two hundred $(\mathrm{N}=1256)$ to identify the substances users a 24-item substance abuse identification inventory was given to the subjects to fill in, out of which one hundred eighty five (185) students were identified as substance users who ticked more than five items for male subjects and three for female subjects. One hundred and five (N-105) (male 54 and female 51) subjects consented to participate in the study.

The research instrument used for this study was a 24 -item substance abuse identification inventory this instrument is used to identify those students that are involved in substance abuse from the list of the items the students were asked to tick $(/)$ and a 25 -item self-developed questionnaire titled Health Education Substance Abuse Intervention Questionnaire (HESAIQ).

To determine the face and content validity of the instrument the researchers' supervisors and two other experts in Educational Measurement and Evaluation from the Departments of Education and Physical and Health Education University of Maiduguri were given for validation. The reliability of the instrument was carried out using split-half .This was considered reliable.

A Letter of introduction from the Head of Department Physical and Health Education, University of Maiduguri was obtained by the researcher seeking for permission to conduct the research study. The subjects were assigned to the three experimental groups for the intervention protocol. The Community-based experimental (Group1) had a trained research assistant who is a community leader and Physical and Health Education specialists who served as a resource person and was trained on the intervention protocol and topics. The three groups were briefed separately and were allowed to discuss with the researcher after the discussions. The questionnaire were administered on the first day to the three groups that is to pre-testing the subject with the help of research assistants. The experimental groups met three times a week for two hours for the health education intervention programme for a period of six weeks.

\section{Method of Data Analysis}

The data collected were analysed using descriptive statistics of percentage scores, mean, and standard deviation to answer research questions and inferential statistics of independent sample paired t-test to test the hypothesis 1 at 0.05 levels of significances.

Research Question: What is the effectiveness of community-based health education intervention programme on student's knowledge of substance abuse, reasons, effects of substance abuse at pretest and post-test intervention among students in College of Education Waka-Biu Borno State Nigeria?

Table 2: Students' knowledge on Substance Abuse, Reasons and Effects taught using Community-based Health Education Intervention

\begin{tabular}{|c|c|c|c|c|c|c|}
\hline \multirow[b]{2}{*}{ Group } & \multirow[b]{2}{*}{ Pretest } & \multicolumn{2}{|c|}{$\begin{array}{l}\text { Knowledge on } \\
\text { substance abuse }\end{array}$} & $\begin{array}{l}\text { Reasons for substance } \\
\text { abuse }\end{array}$ & \multicolumn{2}{|c|}{ Effects of substance abuse } \\
\hline & & Post test & Pretest & Post test & Pretest & Post test \\
\hline & Mean Sd & Mean $\mathrm{Sd}$ & Mean & Mean Sd & Mean Sd & Mean $\mathrm{Sd}$ \\
\hline Community -based & $22.66 \quad 2.30$ & $21.23 \quad 2.04$ & 17.712 .153 & 19.493 .346 & 17.231 .942 & $20.65 \quad 3.317$ \\
\hline
\end{tabular}

Table 4:3 is the summary of mean and standard deviation distribution of pretest and post test scores on knowledge of substance abuse, reasons and effects of substance abuse among students in College of Education Waka-Biu Borno State taught using community-based health education intervention programme.

The result of the studies on knowledge of substance abuse at pretest showed a mean score of $22.66 \pm$ 2.30 and a post-test score of $21.23 \pm 2.04$, this showed decline on knowledge which might be as a result of lack of understanding of what is required, while on the reasons for substance showed a mean score of $17.71 \pm 2.15$ at pretest and post test score of $19.49 \pm 3.34$ and pretest score of $17.23 \pm 1.94$ on the effects of substance abuse and post score of $20.63 \pm 3.31$. The post test result of the subjects showed improvement on its mean score indicating the effectiveness of the community-based intervention.

Hypothesis 1: There is no significant difference between pretest and post test scores of student's knowledge of substance abuse, reasons for substance abuse and effects of substance taught using community-based health education intervention programme.

$$
\mathrm{n}=35
$$




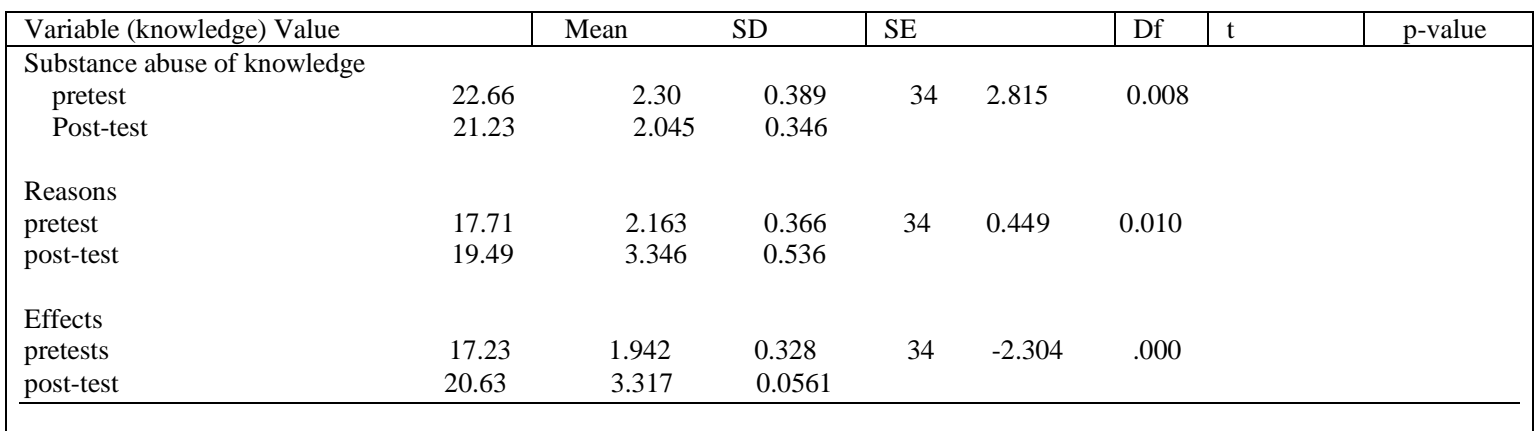

$\mathrm{t}=2.816 ;-0.449, \&-2.304 \mathrm{p}>0.05$

Table 4.9 is the summary of paired sample t-test comparing pretest and post test means score on the effectiveness of community based health education intervention programme on knowledge of substance abuse, reasons and effects of substance abuse. The post test result on knowledge of substance abuse is greater than the pretest of $(22.66 \pm 2.30)>21.23+2.045$. Meaning there is gain on knowledge of substance abuse at post-test and the post-test scores on reasons and effects of substance abuse are greater than the pretest score $(17.71 \pm 2.163>19.49 \pm 3.346)$ and $(17.23 \pm 1.942>20.63 \pm 3.317)$. Meaning that there is gain on the knowledge of reasons and effects of substance abuse among the subjects after the application of community-based health education intervention programme. The result on the table showed that there is a significant difference in the effectiveness of community-based intervention programme on knowledge of substance abuse, and between pretest and post-test score $\mathrm{t}=2.815 \mathrm{df}=34, \mathrm{P}, 0.05$. The table showed $\mathrm{P}$-valve is .008 which is less than the a-valve (0.05), therefore the null hypothesis was rejected. The result on table 4.9 showed that there is a significant difference on the effectiveness of Community-based health education intervention on reasons for substance abuse between pre and post-test scores. $(\mathrm{t}=-0.449$, df $34, \mathrm{P}<0.05)$. The table showed that $\mathrm{P}$ valve which is 0.010 is less than the a-valve (0.05). Therefore, the null hypothesis was rejected. Furthermore P. value on knowledge of effects of substance abuse is 0.000 which is less than a-value (0.05) therefore the mull hypothesis was rejected. Hence, therefore there is no significant difference on the effectiveness of community-based health education intervention programme on knowledge of the effects of substance abuse between pretest and post test $(\mathrm{t}-2.304 \mathrm{df}=34>0.057)$. Therefore, the null hypothesis is hereby retained.

Table 3: Summary of Paired Independent Sample t-test on the Effectiveness of Community-based Health Education Intervention at Pretest and Post-test

\begin{tabular}{|l|cc|c|c|c|c|}
\hline $\begin{array}{l}\text { Variable (knowledge of substance } \\
\text { abuse) }\end{array}$ & Mean Sd & SE & Df & T & p-value \\
\hline Pretest & 57.600 & 4.8816 & 0.82625 & 34 & -3.199 & 0.003 \\
\hline Post test & 62.171 & 6.2284 & 1.05280 & 34 & & \\
\hline
\end{tabular}

Table 3: contains the summary of paired independent sample t-test comparing pretest and post test mean scores on the effectiveness of community-based health education intervention protocol on substance abuse knowledge among students. The post-test means scores of $62.1714 \pm 6.2282>57.60 \pm 4.8816$ on substance abuse knowledge is greater than the pretest scores. Meaning there is positive gain on knowledge of substance abuse indicating the effectiveness of community-based intervention. The result on the table showed that there is a significant improvement on the substance abuse knowledge with a $(\mathrm{t}-=-3.199)$. The $\mathrm{P}$ value calculated is 0.003 which is less than a value (0.05). Therefore, the null hypothesis in rejected.

\section{Finding}

Significant difference exists between pretest and post-test scores on the effectiveness of community-based health education intervention programme on knowledge of substance abuse. Knowledge of the effects of substance abuse at post-test scores is higher than the pretest which indicates that the intervention is effective.

\section{Discussion}

School based health education intervention protocol success in this study could be attributed to the fact that the school in the ideal place that help shape the health and personality of students ( Moronkola1993).The school has the most effective medium to maintain health and wellbeing of students though behavioural modification (Baba etal 2011). in line with this study Ennett, Haws, Ringwatt, Vincus; Hemly, Bowling and Rohrbach (2011) stated that school-based health education intervention are effective since the school has the highest population of youths which can be reached. Roonal et al (2009), Ferguson, San Miguel, Kilburn and 
Sankhez (2007)) asserted that examining the effectiveness of school- based health interventions intended to prevent substance abuse have led to the divergence of opinion about how well different types of intervention would work and for whom. Effective school based substance education programmes that target all students at a particular grade level or series of grade levels are seen by some as an ideal prevention intervention, despite the relatively modest effects that result from trying to prevent substance abuse in a heterogeneous population, that includes many who are predisposed to use or abuse of substance because such programmes are relatively easy to implement and relatively in expensive when opportunity cost are taken into account and effectiveness was observed.

Findings of hypothesis one significant differences exist on knowledge of substance abuse, reasons for substance abuse and effects of substance abuse using community-based health education intervention. This study revealed significant differences on knowledge of substance abuse, while on reasons and effects of substance abused significant gain was observed which indicated significant difference exists on substance abuse knowledge, this findings agreed with Presely et al (1996) in which community-based intervention programme was organized to support reduction of alcohol abuse by adolescent the study reported effectiveness of the intervention protocol in which improvement substance abuse knowledge was gained and resulted in the reduction of the alcohol intake among the subjects. This also agreed with Holder et al (2000) that examined the effectiveness of community-based intervention and found that this intervention resulted into $49 \%$ reduction in drunkenness $10 \%$ reduction in traffic accident and $43 \%$ reduction in first aid and accidents. This finding further agreed with the findings of Rose and Degong (2008) who posit that community-based health education intervention programmes provides the students with the best information, knowledge and skills on substance abuse in which youths can use to safeguard themselves against risk behaviors.

Based on the findings of this study it was concluded that:

\section{Conclusion}

Six weeks of health education intervention programmes improve students' knowledge on substance abuse. Six weeks of interventions taught using community-based, peer-led, and school-based health education intervention progamme is effective in improving knowledge on substance abuse among students in College of Education Waka-Biu. Different methods of intervention prorammes which include community-based, peer-led and school-based intervention programmes are better than others in their effectiveness in improving knowledge of substance abuse. School-based health education intervention programme is the most effective in improving knowledge of substance abuse and showing changes regarding substance abuse. The community-based health education intervention programme used in resolving social issues is least effective in improving knowledge of substance abuse.

\section{Recommendations}

Based on the result of this study, the following recommendations were made.

(1)School-based health education intervention programmes should be adapted to effectively enhanced students substance abuse knowledge.

(2) Different substance abuse health education intervention package to be taught using community-based, peerled, and school-based approaches should be developed, enhance and adapted for utilization in schools.

\section{References}

[1] Adamu, A. (1995). Some Basic Issues on Drug Abuse. A counseling Perspective the Farufaru. Journal of Multi-Displinary Studies 4(1-2): $1-10$

[2] Baba, D. A, Shehu, R.A. \& Oniyabgu, S. O (2011). Impact of School Health Programs on the Health of Primary School Pupils in Moro Local Government Kwara State.

[3] Bajracharya, S. M. (2009). Community-based Health Education Intervention: A Service-Learning Approach. Forum on Public Policy.

[4] Biglan, A, Ary, D. V, Smolkowski, K, Duncan,T. \& Black, C (2000). A Randomized Controlled Trial of a Community Intervention to Prevent Adolescent Tobacco Control. America Psychology Association 9: (24-32)

[5] Chou, C. P, Montgomery, S, Pentz, M.A, Robrbach, L.A, Jonson, C.A, Flay, B.R, \& Mackinnon, D. P (1998). Effect of a Community-Based Prevention Programmed in Decreasing Drug Use in High-Risk Adolescents. American Journal of Public Health:88; (984-8)

[6] Eneh, A.U. \&Stanly P.C (2004) Patten of Substance use among secondary school students in River State, Nigeria journal of Medicine 13(1) 20-24

[7] Ennatte, S.T, Hawst, S, Ringwalt, C.H, Vincus, A.A, Hamly, S, Bawling, J.M \& Rohback, L.A (2011).Evidence-Based Practice in School Substance Use Prevention Fidelity of Implementation Under Real- World Conduction Health Education Theory and Practice; 26(2): 361-371

[8] Ferguson C. J, San Migual .C, Kilburn C. J \& Sankhez. P (2007). The Effectiveness of School-Based Anti-bully programmes; A Mate-Analytic Reviews: Criminal Justice Reviews 32(4): 401-414.

[9] Garba, H. \& Gaya M. W. U. (2005). Incidence of Drug Abuse Among Tertiary Institution Students in Borno State. Journal of Health, Education and Sports Science.5(1);43-55. 
[10] Hyelasindika,S and Mbaya, M.M.(2004) Causes and Effect of Drug Abuse Among Secondary School Students in Bama Local Government Area Borno State. Educational Forum A journal of Educational Studies 7 92-101.

[11] Holder, H.D. Gruenewald, P.J, Ponicki, W,R, Treno, A.J, Grube. J, W, Saltze, R. F, Voas, R.B, Reynolds, R, Davis, J. Sanchez, C, Gaumont G. \& Roefer (2000). Effect of Community-Based Intervention on High Risk Drug and Alcohol Related Injuries. Journal of the American Medical Association 284: 2341-7

[12] Makanjuola, A.B, Daramola, T.O \& Obembe, A. O (2007). Psychoactive Substance Use Among Medical Students in a Nigerian University. World Psychiatry; 6 (2):48-50

[13] Presely, C. A, Meilaman, P.W \& Cashin, J. R (1996). Alcohol and drug on American college campuses; use, consequences and perception of the campus environment, vol. iv, 1992-1994 (Carbondale,iii: core institute, southern lllions University).

[14] Racah, B. \& Soares, R, R. (2009). Evaluating the Impact of Community-based Health Education Intervention:- Evidence from Brazils family Health Program Discussion 184119

[15] Rose, V \& Degong, W (2008). Alcohol and Other Drug Abuse Among First-year College Students V.S.A of Department of Education.

[16] Roonal, M. Streke A; Ochshorn, P. \& Palmer, A (2009). Identifying Effectiveness School-based Substance Abuse Prevention Intervention. New York: Haworth Press.

[17] Stelkler A, Altman J. P, Brawn R, Burnadure W. J, Goodman R. M, \& Forgersen .R. M (2012) Education Intervention Strategies: Recommendation for Future Research Abstract.

[18] Wandersman, A, \& Florini, P. (2003). Community Intervention and Effective Prevention

Garba. "Community-Based Health Education Intervention Programe On Substance Abuse Among College Of Education Students In Nigeria." IOSR Journal of Nursing and Health Science (IOSR-JNHS) 6.4 (2017): 58-63. 